\title{
THE SOURCE JERK INTEGRAL METHOD FOR SUB-CRITICALITY MEASUREMENTS IN ADS
}

\author{
A. Kochetkov ${ }^{1}$, A. Billebaud ${ }^{2}$, S. Chabod ${ }^{2}$, A. Krása ${ }^{1}$, F.-R. Lecolley ${ }^{3}$, J.-L. Lecouey ${ }^{3}$, \\ G. Lehaut ${ }^{3}$, N. Marie $^{3}$, N. Messaoudi ${ }^{1}$, G. Vittiglio ${ }^{1}$ and J. Wagemans ${ }^{1}$ \\ ${ }^{1} \mathrm{SCK} \cdot \mathrm{CEN}$, Belgian Nuclear Research Centre \\ Boeretang 200, 2400 Mol, Belgium \\ ${ }^{2}$ LPSC, Université Grenoble-Alpes, CNRS/IN2P3 \\ 53 Avenue des Martyrs. 38026 Grenoble Cedex, France \\ ${ }^{3}$ Normandie Univ, ENSICAEN, UNICAEN, CNRS/IN2P3 \\ LPC Caen, 14000 Caen, France \\ akochetk@sckcen.be
}

\begin{abstract}
Three sub-critical (SC) core configurations were investigated in the VENUS-F zero power reactor coupled with the GENEPI-3C accelerator. The SC10 and SC12 were a mock-up of a MYRRHA start-up core and SC11 represented a more complex MYRRHA core loaded with various types of in-pile-sections. The sub-criticality of 11 variants of these VENUS-F cores was changed in several steps from $-6 \$$ down to $-30 \$$ using the safety and control rods. Their sub-criticalities were determined with the Source Jerk Integral (SJI) method using 11 fission chambers located all over the reactor. For the data analysis, the 8-group delayed neutron parameters from the JEFF-3.1.2 evaluated nuclear data library were used. Reliability and reproducibility of the experimental results were tested by repeating the measurements, swapping the detectors and varying the accelerator beam intensity, thus changing the detector count rates and verifying the validity of the dead time corrections. The obtained results are compared with MCNP calculations.
\end{abstract}

KEYWORDS: zero-power ADS experiments, source jerk method

\section{INTRODUCTION}

Accurate sub-criticality measurements of ADS needed to be demonstrated for the licensing of this type of installations. During the past 20 years several European projects were launched to develop a reliable on-line subcriticality monitoring method. These projects were MUSE FP5 [1] in the MASURCA zero power facility (France) coupled with the GENEPI pulsed neutron source, and GUINEVERE in EUROTRANS-IP FP6 [2] and FREYA FP7 [3] in the VENUS-F zero power facility (Belgium) coupled with the GENEPI-3C pulsed/continuous neutron source. During the MUSE project it was observed that most of the well-known methods used for sub-criticality determination (pulsed neutron source, all noise methods) cannot be applied for the industrial ADS machines like MYRRHA [4] due to the continuous beam of the accelerator or the rather deep level of core sub-criticality $\left(\mathrm{k}_{\mathrm{eff}} \approx 0.95\right)$. The only absolute method identified as an appropriate candidate for the on-line sub-criticality monitoring in the MYRRHA ADS is the source jerk method during 
a beam interruption (BI) [5] of the accelerator operation. This method was verified in the MYRTE H2020 project [6]. Then, three sub-critical VENUS-F core configurations were investigated: SC10 and SC12, serving as a mock-up of a MYRRHA start-up (homogeneous) core and SC11 representing a complex MYRRHA core loaded with various types of in-pile-sections (IPS).

To verify the applicability and reliability of this method, a reference method has to be used too. Since the MUSE experiments till nowadays the classical rod drop-MSM method [7] is considered as the reference. This method requires an initial critical core which can be easily realized in a zero power facility. However the results of this method have a strong dependence on the position of the detectors and calculated correction factors are needed to get the final results [5]. As an alternative technique we present here the Source Jerk Integral (SJI) method that was applied during the FREYA and MYRTE experiments. It works on the principle of a sudden stop of the neutron source, after which the complete delayed neutron population is recorded. Its main advantages are that it is less dependent on the position of the detectors and does not require any reference to a critical state (on contrary to the MSM method). The main disadvantage of the SJI method is that the beam interruption has to be long enough to record the complete decay of the delayed neutrons $\left(\approx 15 \mathrm{~min}\right.$ at VENUS-F for $\left.\mathrm{k}_{\mathrm{eff}}=0.95\right)$ which then prevents it to be used for online sub-criticality monitoring during the reactor operation. However, the SJI method can be considered as an alternative method for the sub-criticality measurements during the core loading phase where it could be possible to start and stop the proton beam just for the purpose of the sub-criticality level determination.

The sub-criticality of the SC10, SC11 and SC12 VENUS-F cores coupled with the GENEPI-3C accelerator was changed in several steps from $-6 \$$ down to $-30 \$$ using the safety and control rods. The reactivity level of all variants were determined with SJI method. The obtained results are compared with MCNP calculations.

\section{VENUS-F AND GENEPI-3C ZERO POWER ADS}

A fast lead/lead-bismuth VENUS-F core coupled with the GENEPI-3C deuteron accelerator is used as the zero power ADS system at SCK $• \mathrm{CEN}$ since 2011. The facility is used for experiments related to subcriticality monitoring method tests and nuclear data validations for MYRRHA and fast neutron systems cooled with liquid heavy metal.

The VENUS-F reactor consists of a Stainless Steel (SS) square casing that is inserted in the cylindrical vessel of the previous VENUS-T water moderated reactor. This SS casing can be filled with $144(12 \times 12)$ square assemblies $(8 \times 8 \mathrm{~cm})$. In turn, the assemblies can be filled with round or square rodlets of metallic uranium (30 wt\% enriched), lead, bismuth, alumina or graphite and lead blocks. The SS casing also comprises 6 safety and 2 control rods (SR and CR respectively) and a dozen of reflector assemblies with holes for insertion of detectors. The height of the core is $60 \mathrm{~cm}$. Around the core there are $40 \mathrm{~cm}$ top and bottom lead reflectors, as well as a radial reflector around the casing, filling the whole $160 \mathrm{~cm}$ diameter of the VENUS vessel. The details of the VENUS-F reactor can be found in [8] and Figures 1-2. When the reactor is in a sub-critical configuration, the four central assemblies are replaced by the GENEPI-3C beam tube ended by the TiT target.

The external neutron source is provided by the GENEPI-3C deuteron accelerator coupled to a tritiated titanium target located at the core mid-plane of the VENUS-F reactor. GENEPI-3C accelerates deuterons up to $220 \mathrm{keV}$. Their interaction with the TiT target mainly generates a quasi-isotropic field of $\sim 14 \mathrm{MeV}$ neutrons through $\mathrm{T}(\mathrm{d}, \mathrm{n})^{4} \mathrm{He}$ fusion reactions [9]. Three modes are available for the operation of the accelerator: pulsed mode, continuous mode and continuous mode with short beam interruptions. In the work presented here, the last two were used. 


\section{SUB-CRITICAL CONFIGURATIONS}

In this work, two main sub-critical VENUS-F core configurations are discussed: SC10 (Figure 1) serving as a mock-up of a MYRRHA start-up "clean" core and SC11 (Figure 2) representing a complex MYRRHA core loaded with two types of in-pile-sections (IPS). One type of IPS simulated empty (Mo-2) and filled (Mo-1) thermal spectrum molybdenum production units. These are mainly composed of polyethylene, cadmium, with a fuel rodlet in addition for the filled IPS. The other type is made of SS, to simulate a MYRRHA material testing IPS. The position $(-6,-2)$ in the cores was occupied with a rod drop system having a small reactivity absorber rod called POAR (POwder Absorber Rod) that can be dropped into the core and which served for the MSM measurements [8]. Finally, the core was surrounded by a ring of graphite assemblies to simulate the $\mathrm{BeO}$ radial reflector of MYRRHA.

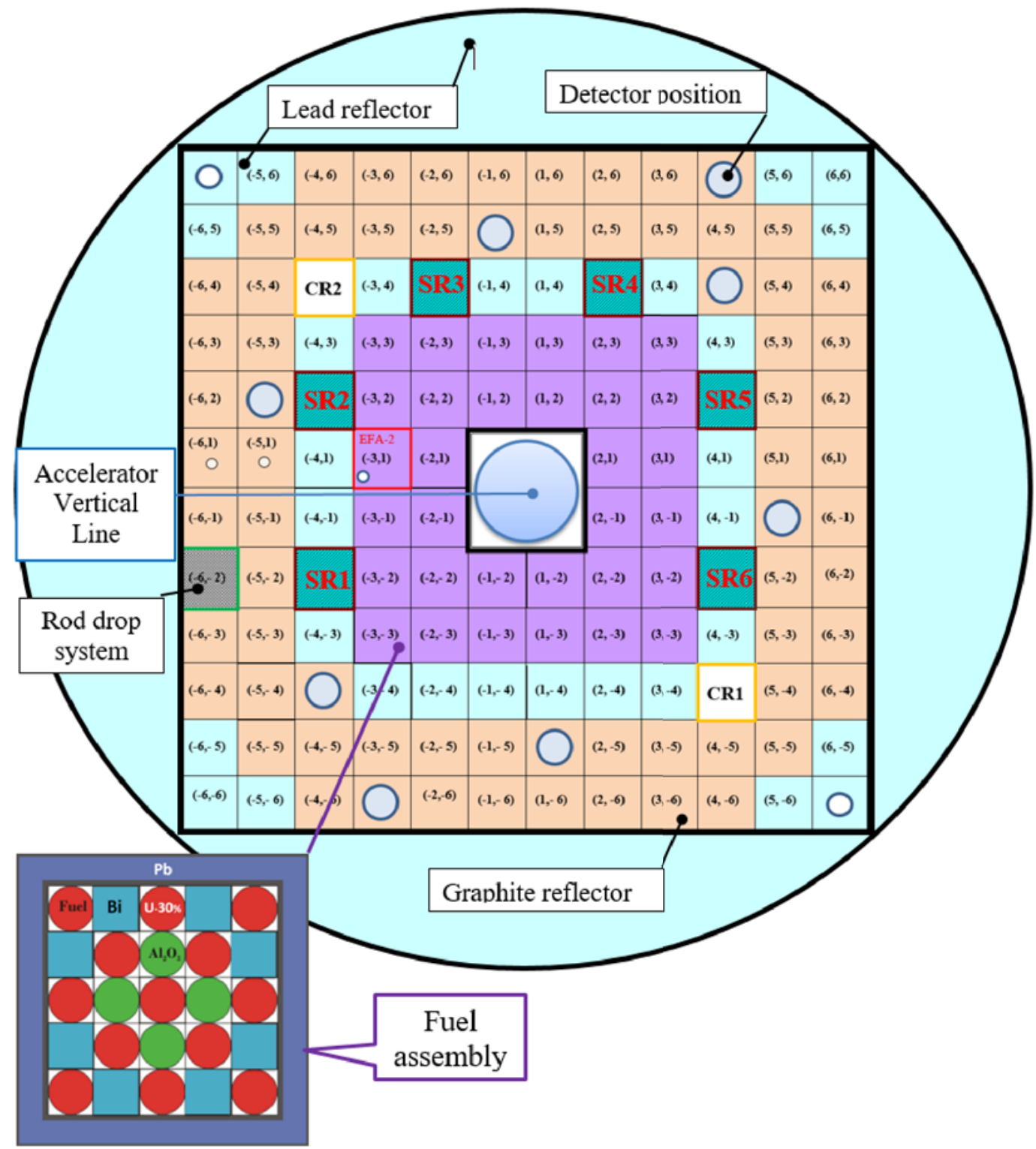

Figure 1. The SC10 sub-critical core layout. 


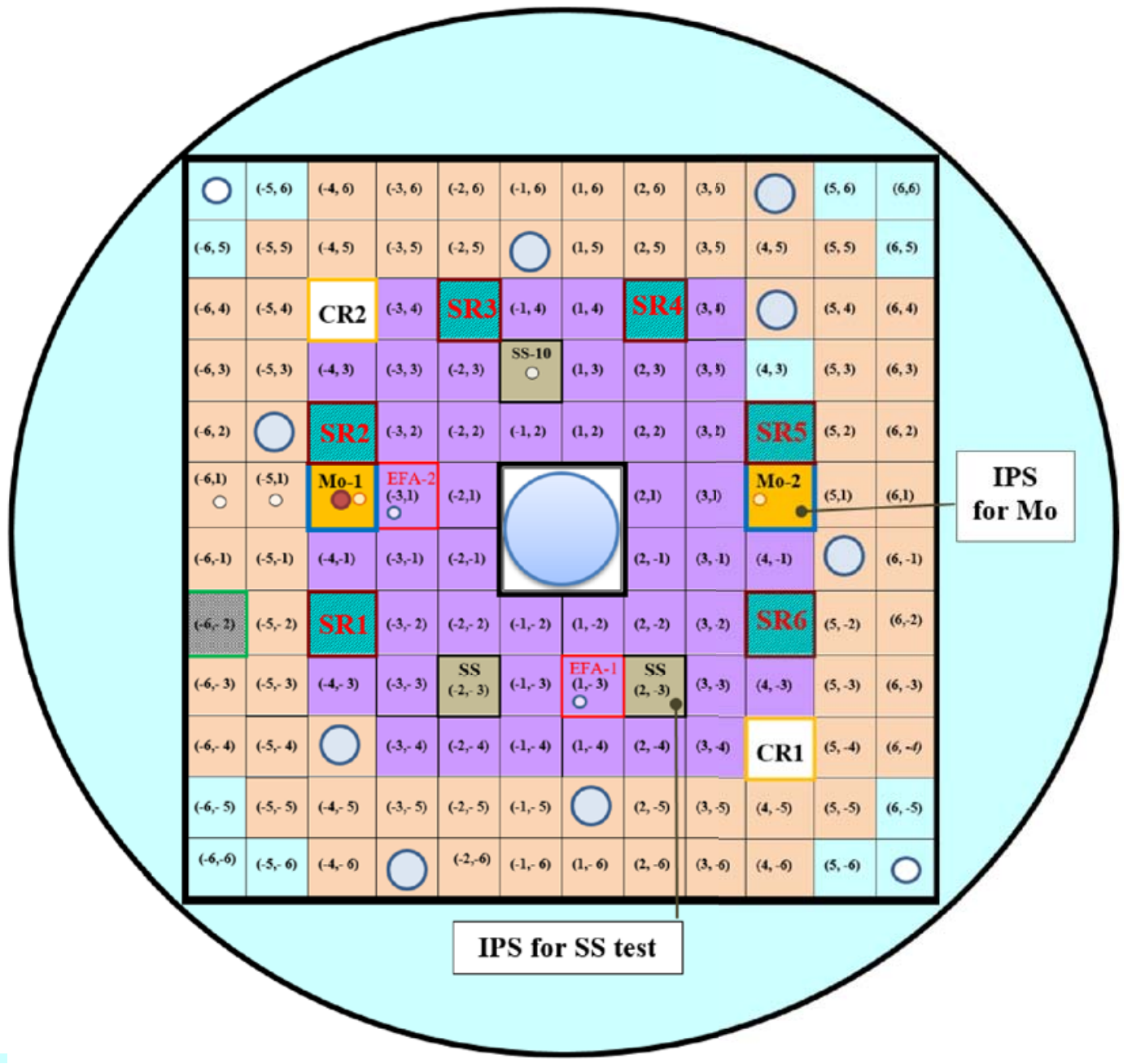

Figure 2. The SC11 sub-critical core layout.

The SC12 core was the same as the SC10 one but without 2 fuel assemblies (in positions $(3,3)$ and $(-3,-3)$ ), which were replaced with $\mathrm{Pb}$ reflector assemblies to have a deeper sub-criticality.

The sub-criticality level of the investigated cores were varied by changing the positions of the SRs and CRs. All studied configurations are listed in Table I. The CR position indicating $600 \mathrm{~mm}$ means the rod is fully withdrawn, and $0 \mathrm{~mm}$ fully inserted. The $361.5 \mathrm{~mm}$ position represents the height of the rods when the core was critical, without accelerator vertical line in the center. The results of $k_{\text {eff }}$ that are presented in Table I were obtained with MCNP calculations. The details of the calculation tools are presented and discussed further.

\section{NEUTRONIC CALCULATION TOOLS: CODES AND NUCLEAR DATA}

The Monte Carlo calculations of $\mathrm{k}_{\text {eff }}$ and spectrum averaged delayed neutron parameters were obtained with the MCNP5-1.60 code [10] using the JEFF-3.1.2 nuclear data library [11] that considers 8-group of delayed neutron parameters [12]. 
Table I. The SC10, SC11 and SC12 core variants that have been studied.

\begin{tabular}{|c|c|c|c|c|c|}
\hline Core & $\begin{array}{l}\text { Number of } \\
\text { SRs IN }\end{array}$ & $\begin{array}{c}\text { CRs height, } \\
(\mathrm{mm})\end{array}$ & $\begin{array}{c}\text { POAR } \\
\text { Position } \\
\end{array}$ & $\mathrm{k}_{\mathrm{eff}}, \mathrm{MCNP}$ & $\begin{array}{c}\boldsymbol{\beta}_{\text {eff, }}, \mathbf{M C N P}, \\
\text { pcm }\end{array}$ \\
\hline SC10 \0 & 0 & 600 & OUT & $0.95169(2)^{*}$ & $737(3)$ \\
\hline$S C 10 \backslash 4$ & 4 & 0 & IN & $0.86663(1)$ & $739(2)$ \\
\hline$S C 10 \backslash 6$ & 6 & 0 & IN & $0.83700(1)$ & $747(2)$ \\
\hline SC11 & 0 & 361.5 & IN & $0.96282(2)$ & $730(2)$ \\
\hline SC11\0 & 0 & 0 & IN & $0.95506(4)$ & $730(2)$ \\
\hline SC11\2 & 2 & 0 & IN & $0.92155(2)$ & $732(2)$ \\
\hline SC11\4 & 4 & 0 & IN & $0.89878(2)$ & $731(2)$ \\
\hline SC11\6 & 6 & 0 & $\mathrm{IN}$ & $0.87511(2)$ & $736(2)$ \\
\hline SC12\0 & 0 & 600 & OUT & $0.93669(1)$ & $741(2)$ \\
\hline SC12\2 & 2 & 0 & IN & $0.88997(1)$ & $748(2)$ \\
\hline SC12\6 & 6 & 0 & IN & $0.82281(2)$ & $746(2)$ \\
\hline
\end{tabular}

* ( ) - statistic errors

For each multiplication factor calculation, $1 \times 10^{6}$ particles in at least 1700 cycles were used and statistical tests were performed to make sure to yield sufficiently small uncertainties $(\sigma)$ on the kinetic parameters. 50 inactive cycles were needed to ensure the fission source convergence.

\section{SOURCE JERK INTEGRAL METHOD}

The source jerk method is a well-known method [13] based on rapidly taking out a neutron source from a subcritical reactor core. Its so-called "integral" variant is applied in the VENUS-F sub-critical experiments since 2012 [14].

According to this method, deduced from point kinetics, to get the negative reactivity of the core in $\$$ we need the count rate from a detector averaged over a duration of about 1000 seconds before the source jerk $\left(\mathrm{N}_{0}=\right.$ counts per second) when the reactor is at a stable power, and the integral of the counts $(\mathrm{N}(\mathrm{t}))$ from the time $t_{0}$ of the source jerk till the end of the delayed neutron emission process (theoretically zero counts in the detector):

$$
\frac{\rho}{\beta_{e f f}}=-\left[N_{0} * \sum_{i=1}^{8} \frac{a_{i}^{e f f}}{\lambda_{i}}\right] / \int_{\mathrm{t} 0}^{\infty} N(t) d t,
$$

Where:

$a_{i}{ }^{e f f}=\beta_{i} / \beta_{\text {eff, }}$, the fraction of the delayed neutrons of group $i$ (relative yields, 8 groups in our case).

$\lambda_{i}-8$ groups decay constants.

$\mathrm{N}_{0}$ - count rate from the detector when the GENEPI-3C source is on.

$\mathrm{N}(\mathrm{t})$-count rate from the detector as a function of time after GENEPI-3C is switched off.

The delayed neutron parameters used for the analysis were calculated with the MCNP code. It gives $\beta_{\text {eff }}$ values ranging from 730 to $748 \pm 2 \mathrm{pcm}$, depending on the core variant (see Table I). For the each core configuration the appropriate $\beta_{\text {eff }}$ with the corresponding $\mathrm{a}_{\mathrm{i}}{ }^{\text {eff }}$ were used. 


\section{RESULTS OF THE MEASUREMENTS}

Fission chambers with ${ }^{235} \mathrm{U}$ and ${ }^{238} \mathrm{U}$ deposits with masses ranging from 1 to $1000 \mathrm{mg}$ were used for the measurements (see Table II). They were placed in the lead reflector, the graphite reflector and the fuel zone. To test the reproducibility of the method, different detector sets were used for the source jerk experiments in each sub-critical core on different days, months and sometimes years. For a same configuration the reactivity values obtained with the SJI method in different time always were consistent (standard deviations are in the range $0.1-0.7 \%$ when averaging, the details will be in presentation).

In the experiments, the beam current of the accelerator in continuous mode (and then the neutron source) is varied from 40 to $500 \mu \mathrm{A}$. This variation led sometimes to poor statistics in the detectors having a light or threshold deposit mass (see the digits in red in the last column of Table II). The results of some detectors were then omitted from the average. In any case each configuration reactivity value was obtained by averaging at least six detector results (see example for SC10/4 core in Table II and Figure 3).

Table II. Reactivity values obtained in SC10/4 core with detector Setup E.

\begin{tabular}{|c|c|c|c|c|c|c|}
\hline \# Det & Name & Main deposit & Mass $(\mathrm{mg})$ & Position & $\rho(\$)$ & \pm stat, \% \\
\hline D1 & RS-75 & ${ }^{235} \mathrm{U}$ & 110 & $(-6,6)$ & -21.81 & 0.77 \\
\hline D2 & RS-72 & ${ }^{235} \mathrm{U}$ & 110 & $(-3,-6)$ & -21.85 & 0.44 \\
\hline D3 & RS-79 & ${ }^{235} \mathrm{U}$ & 110 & $(5,-1)$ & -21.25 & 0.40 \\
\hline D4 & CFUL676 & ${ }^{238} \mathrm{U}$ & 1150 & $(-4,-4)$ & -23.72 & 3.24 \\
\hline D5 & CFUL673 & ${ }^{238} \mathrm{U}$ & 1100 & $(4,4)$ & -24.73 & 5.50 \\
\hline D6 & CFUM668 & ${ }^{235} \mathrm{U}$ & 10 & $(-1,5)$ & -22.04 & 1.73 \\
\hline D7 & CFUM667 & ${ }^{235} \mathrm{U}$ & 10 & $(1,-5)$ & -21.19 & 1.22 \\
\hline D8 & CFUF34 & ${ }^{235} \mathrm{U}$ & 1 & $(-3,1)$ & -23.74 & 8.06 \\
\hline D9 & RS-71 & ${ }^{235} \mathrm{U}$ & 110 & $(4,6)$ & -22.00 & 0.49 \\
\hline D10 & CFUL677 & ${ }^{238} \mathrm{U}$ & 1150 & $(-5,2)$ & -22.71 & 2.32 \\
\hline D11 & RS-74 & ${ }^{235} \mathrm{U}$ & 110 & $(6,-6)$ & -21.24 & 0.69 \\
\hline
\end{tabular}

The results of the sub-criticalities measured with the SJI method are compared with calculations obtained with MCNP in Table III. The total uncertainty on the measured $\rho / \beta$ values has been calculated applying standard error propagation and include dead time correction errors on $\mathrm{N}_{0}$, the statistical uncertainty on the integral count rates and the uncertainty on the delayed neutron data a(i), $\lambda(i)$ used in equation (1). The uncertainty on the MCNP calculations include the uncertainty on the $k_{\text {eff }}$ and $\beta_{\text {eff }}$ calculations. 


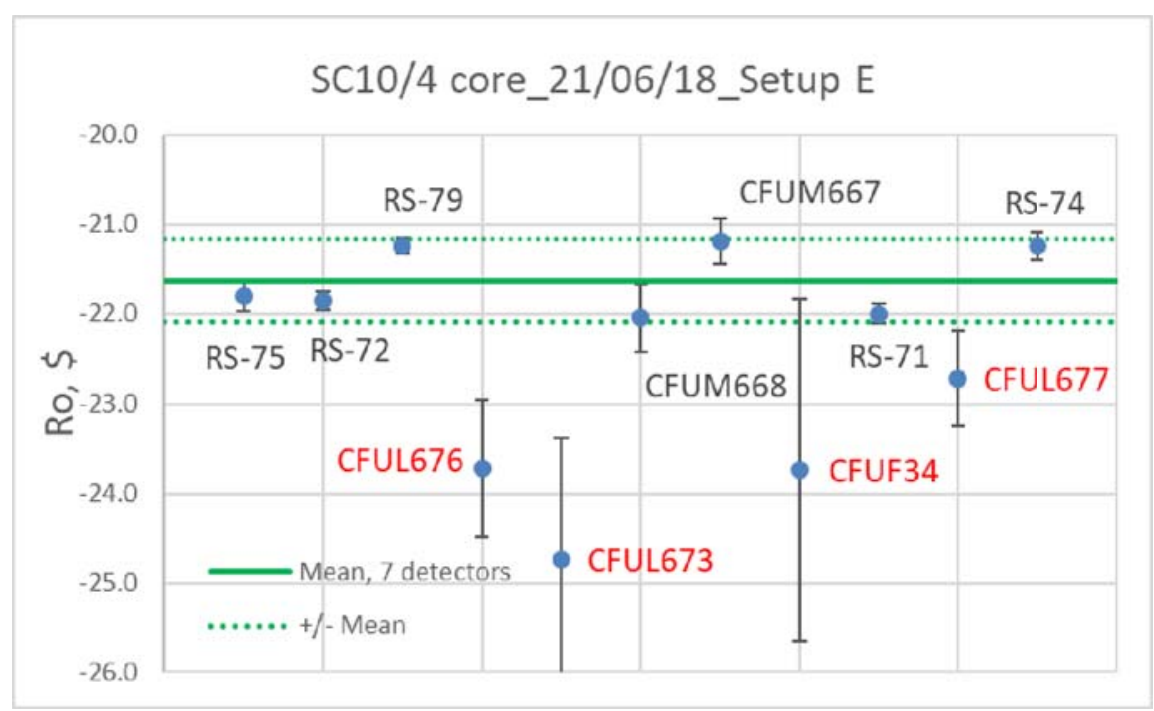

Figure 3. The SC10/4 core sub-criticality measured with detector Setup E.

Table III. Reactivity values obtained with SJI method and MCNP calculations for configurations.

\begin{tabular}{|c|c|c|c|c|c|c|}
\hline Core & \multicolumn{2}{|c|}{ Experiment } & \multicolumn{2}{|c|}{ MCNP } & \multicolumn{2}{|c|}{ MCNP/Experiment } \\
\hline Variant & $\rho,(\$)$ & $\pm \mathbf{a b s}$ & $\rho,(\$)$ & $\pm \mathbf{a b s}$ & $1-C / E$ & \pm \\
\hline SC10 $\backslash 0$ & -7.76 & 0.16 & -6.89 & 0.03 & $11.2 \%$ & $2.1 \%$ \\
\hline $\mathrm{SC} 10 \backslash 4$ & -21.65 & 0.46 & -20.82 & 0.06 & $3.8 \%$ & $2.1 \%$ \\
\hline SC10\6 & -26.83 & 0.67 & -26.07 & 0.07 & $2.8 \%$ & $2.5 \%$ \\
\hline SC11 & -5.80 & 0.12 & -5.29 & 0.01 & $8.7 \%$ & $2.1 \%$ \\
\hline SC11】0 & -6.98 & 0.15 & -6.45 & 0.02 & $7.6 \%$ & $2.1 \%$ \\
\hline SC11】2 & -12.05 & 0.25 & -11.63 & 0.03 & $3.5 \%$ & $2.1 \%$ \\
\hline SC11\4 & -15.90 & 0.33 & -15.42 & 0.03 & $3.0 \%$ & $2.1 \%$ \\
\hline SC11\6 & -20.17 & 0.42 & -19.39 & 0.05 & $3.9 \%$ & $2.1 \%$ \\
\hline SC12\0 & -10.13 & 0.21 & -9.12 & 0.02 & $9.9 \%$ & $2.1 \%$ \\
\hline SC12\2 & -17.54 & 0.37 & -16.53 & 0.04 & $5.8 \%$ & $2.1 \%$ \\
\hline $\mathrm{SC12 \backslash 6}$ & -30.27 & 0.64 & -28.87 & 0.08 & $4.6 \%$ & $2.1 \%$ \\
\hline
\end{tabular}

\section{CONCLUSIONS}

The reactivity of eleven VENUS-F sub-critical configurations simulating different MYRRHA core variants was measured with the SJI method in the period 2017-2019. The method showed stable results with the source intensity and detector position variations. Because of the page limit of the papers, these results will be shown in the presentation. The difference in $\mathrm{k}_{\text {eff }}$ with MCNP values is higher than the uncertainties and is ranging from 400 to $700 \mathrm{pcm}$. This is of the same order of magnitude as the difference observed in the previous VENUS-F critical cores [15], where the MCNP overestimation was between 480 and $1060 \mathrm{pcm}$ depending on the nuclear data library. The reactivities of the cores presented here were also measured with the MSM and BI methods. The analysis is ongoing. 


\section{ACKNOWLEDGMENTS}

This work was partially supported by the H2020 Framework of the European Commission (EURATOM) through the MYRTE project (contract \# 662186).

The authors would like to express their gratitude to the VENUS-F and GENEPI-3C technical teams for their invaluable work during the experiments.

\section{REFERENCES}

1. R. Soule et al., "Neutronic studies in support of accelerator-driven systems: The MUSE experiments in the MASURCA facility", Nucl. Sci Eng. 148, pp. 124-152, (2004).

2. P. Baeten et al, "The GUINEVERE project at the VENUS facility", In Proceedings of the International Conference on the Physics of Reactors (PHYSOR-2008), Interlaken, Switzerland, September 14-19 (2008).

3. A. Kochetkov et al., "Current progress and future plans of the FREYA Project", Proc. Int. Conf., Technology and Components of Accelerator Driven Systems (TCADS-2), Nantes, France, May 21-24, pp. 78-87 (2013).

4. D. De Bruyn, H. Aït Abderrahim, P. Baeten and C. Angulo, "The Belgian MYRRHA ADS Programme. Part 1: The new phased implementation plan", Proc. Int. Conf., International Congress on Advances in Nuclear Power Plants (ICAPP'18), Charlotte, North Carolina, USA, pp. 1066-1073 (2018).

5. Lecouey, J. L. et al., "Reactivity measurement with threshold fission chambers in the MYRRHA mockup fast subcritical reactor Venus-F", In Proceedings of the PHYSOR 2018, Cancun, Mexico, April 2226, (2018)

6. A. Kochetkov et al., "Zero power coupling experiments in support of the MYRRHA ADS in the frame of the MYRTE project", In Proceedings of the 3rd International Workshop on Technology and Components of Accelerator-Driven Systems (TCADS-3), Mito, Japan, 6-9th September, pp. 221-233, (2016).

7. Lecouey, J. L. et al., "Estimate of the reactivity of the VENUS-F subcritical configuration using a Monte Carlo MSM method," Annals of Nuclear Energy, 83, pp 65-75 (2015).

8. W. Uyttenhove et al., " Detector positioning for the initial sub-criticality level determination in accelerator driven systems", PHYSOR 2012 Advances in Reactor Physics Linking Research, Industry, and Education, Knoxville, Tennessee, USA, April 15-20, (2012)

9. M. Baylac et al., "The GENEPI-3C Accelerator for the GUINEVERE Project," Proceedings of the International Topical Meeting on Nuclear Research Applications and Utilization of Accelerators (AccApp '09), Vienna, Austria, 4-8 May, (2009).

10. X-5 Monte Carlo Team, "MCNP - A General N-Particle Transport Code, Version 5", LA-UR-03-1987 (2005).

11. A. Santamarina, D. Bernard, Y. Rugama, "The JEFF-3.1.1 Nuclear Data Library", JEFF Report 22 (2009).

12. G.D. Spriggs et al., "An 8-group delayed neutron model based in a consistent set of half-lives", Progress in Nuclear Energy, 41, No.1-4, pp.223-251, (2002).

13. W.S.Hogan, "Negative reactivity measurements", NSE, 8, p.518, (1960)

14. A.Kochetkov et. al., "An alternative Source Jerk method implementation for the subcriticality estimation of the VENUS-F subcritical core in the FREYA Project", In Proceedings of the PHYSOR 2014, Kyoto, Japan, September 28 - October 3, (2014).

15. A. Krása et.al., "Comparative Study on Neutron Data in Integral Experiments of MYRRHA Mockup Critical Cores in the VENUS-F Reactor", EPJ Web of Conferences, ND-2016, 146, 06019, (2017) 